\title{
Non-replaced Mandibular First Molars and Temporomandibular Joint Dysfunction
}

\author{
Gupta SK, ${ }^{1}$ Pratibha PK, ${ }^{1 *}$ Bhat KM, ${ }^{1}$ Mutalik $S,{ }^{2}$ Guddattu $V^{3}$ \\ ${ }^{1}$ Department of Periodontics, ${ }^{2}$ Department of Oral Medicine and Radiology, Manipal College of Dental Sciences, Manipal \\ University, Manipal, Karnataka, India, ${ }^{3}$ Department of Statistics; Manipal University, Manipal, Karnataka, India
}

*Corresponding Author:

Dr. Pratibha PK, MDS

Department of Periodontics

Manipal College of Dental Sciences,

Manipal University, Manipal, Karnataka, India

Email : bg_pratibha@yahoo.co.in

Citation

Gupta SK, Pratibha PK, Bhat KM, Mutalik S, Guddattu V. Non-replaced Mandibular First Molars and Temporomandibular Joint Dysfunction. Nepal Journal of Medical Sciences 2014;3(1):57-62.

\begin{abstract}
Background: Mandibular first molars serve as key teeth of occlusion. Non replacement of these teeth may affect stability of the dentition and have long term effects on the Tempero mandibular joint. The purpose of this study was to observe the association between nonreplaced mandibular molars and temporomandibular joint dysfunction (TMD).
\end{abstract}

Methods: A total of 174 patients, both male and female with missing mandibular first molars, were included in the study. The changes due to non-replacement of mandibular molars were observed clinically. Temporomandibular joint (TMJ) was examined for clinical and radiographic changes. Intra oral findings were correlated with TMJ findings.

Results: Supra eruption of opposing maxillary molars and drift of adjacent teeth had a significant association with asymptomatic clicking of the TMJ. Further, patients with deep bite had radiographic findings of condylar flattening. Clicking of the TMJ was a frequent finding in patients with longer duration of molar loss.

Conclusion: Missing molars not only have periodontal implications but also far reaching effects on the condylar health of the associated TMJ.

Keywords: Missing first molar; occlusion; temporomandibular joint dysfunction

\section{Background:}

Temporomandibular joint (TMJ) is an important hinge of the masticatory apparatus. Its health and well-being very much depends on the occlusion of the dentition. Hence, when the key teeth of occlusion are lost and are not replaced on time, it may lead to disturbed functioning of the TMJ.
The relationship between TMJ and occlusion has always been a matter of discussion and curiosity among all the researchers. It has been suggested by studies that human mandibular condyles can change by functional remodeling in response to changes in occlusion. "Remodeling pattern in TMJ is directly related to changes in occlusal patterns thus 
demonstrating that biological remodeling of the TMJ is a response to the change in functional demands".

Mandibular molars are considered as key teeth of occlusion. Loss of these molars and their non-replacement can bring about a number of changes in the occlusion such as drifting, supraeruption and changes in vertical dimension. However, these changes are seldom noticed and often ignored, perhaps, due to the changes being gradual. Literature review concerning the effects of loss of mandibular molar and its effect on TMJ are few and they present supporting as well as contradicting data. The purpose of this study is to unveil how far apparent the small gradual changes occurring due to the missing key teeth that safeguard the occlusion i.e., the mandibular first molars, are responsible for changes in the TMJ.

\section{Methods:}

\section{Participants and groups}

After obtaining due approval from the Kasturba hospital Ethical committee of Manipal, 174 subjects reporting to the Department of Periodontology, Manipal College of Dental Sciences, Manipal, Karnataka, India, were recruited for the study with their consent. Both male and female subjects in the mean age group of 49 years ( 21 to 80 years) were screened for the missing unilateral and/ or bilateral mandibular first molars. Only those patients who met the following criteria were included in the study:

1. Missing unilateral or bilateral mandibular molars

2. Patients not wearing dentures/prosthetic appliances /orthodontic appliances

3. Patients without a prior history of temporomandibular joint pain and diseases like arthritis

4. Patients without parafunctional habits like bruxism.

\section{Tooth loss recordings}

The findings associated with missing molars were categorized as

1. Shift in tooth position

2. Change in vertical dimension

3. Duration of tooth loss

Shift in tooth position included drifting, supraeruption of the molars /premolars in the opposing arch, pathologic migration, drifting of the adjacent teeth and flaring of the anterior teeth.

Change in vertical dimension included recording of occlusal wear and deep bite.

\section{Temporomandibular joint recordings}

Clinical evaluation: Temporomandibular joint was palpated for clicking and observed clinically for changes like deviation on mouth opening and pain in TMJ joint.

Radiographic evaluation: Orthopantomographs were taken for the patients with the missing mandibular molars. Condylar remodeling as a biologic adaptation was inferred radiographically by checking for condylar flattening. Condylar flattening was noticed radiographically by the evidence of the loss of the superior curvature of the condyle and with loss of radioopacity of the cortical outlines of the condyle. This was done by keeping in mind the condylar asymmetry of the right and left temporomandibular joints. ${ }^{2}$

\section{Bias control}

All the clinical and radiographic examinations were completed by a single examiner and radiographic findings were further confirmed by a specialist in Oral Medicine and Radiology, who was blinded to the results of the clinical examination.

\section{Data analysis}

Frequency and percentages were used to summarize categorical variables. Median and inter quartile range were used to summarize duration of tooth loss. Chi square test was used as a test of association between intra oral variables and presence or absence of clicking and condylar flattening. Mann Whitney' $U$ ' test was used for assessing difference in median duration of tooth loss with presence or absence of clicking, or condylar flattening. Statistical analysis was performed using SPSS version 15.A p value of less than 0.05 was considered to be statistically significant.

\section{Results:}

One hundred seventy four patients, both males and females, examined for the current study were in the age group of 21 to 80 years. Among the factors analyzed, deep bite showed a significant relationship with Temporomandibular joint condylar flattening $(\mathrm{p}<0.05)$. Fifty one patients with deep bite had flattened condyles and 18 patients with deep bite did not exhibit significant changes in the condyle symmetry. Forty nine patients, who did not demonstrate deep bite also showed radiographic changes in the condylar shape. (Table I). 
TABLE I: Summary table of intra oral variables with Flattening of the condyles \&clicking of TMJ

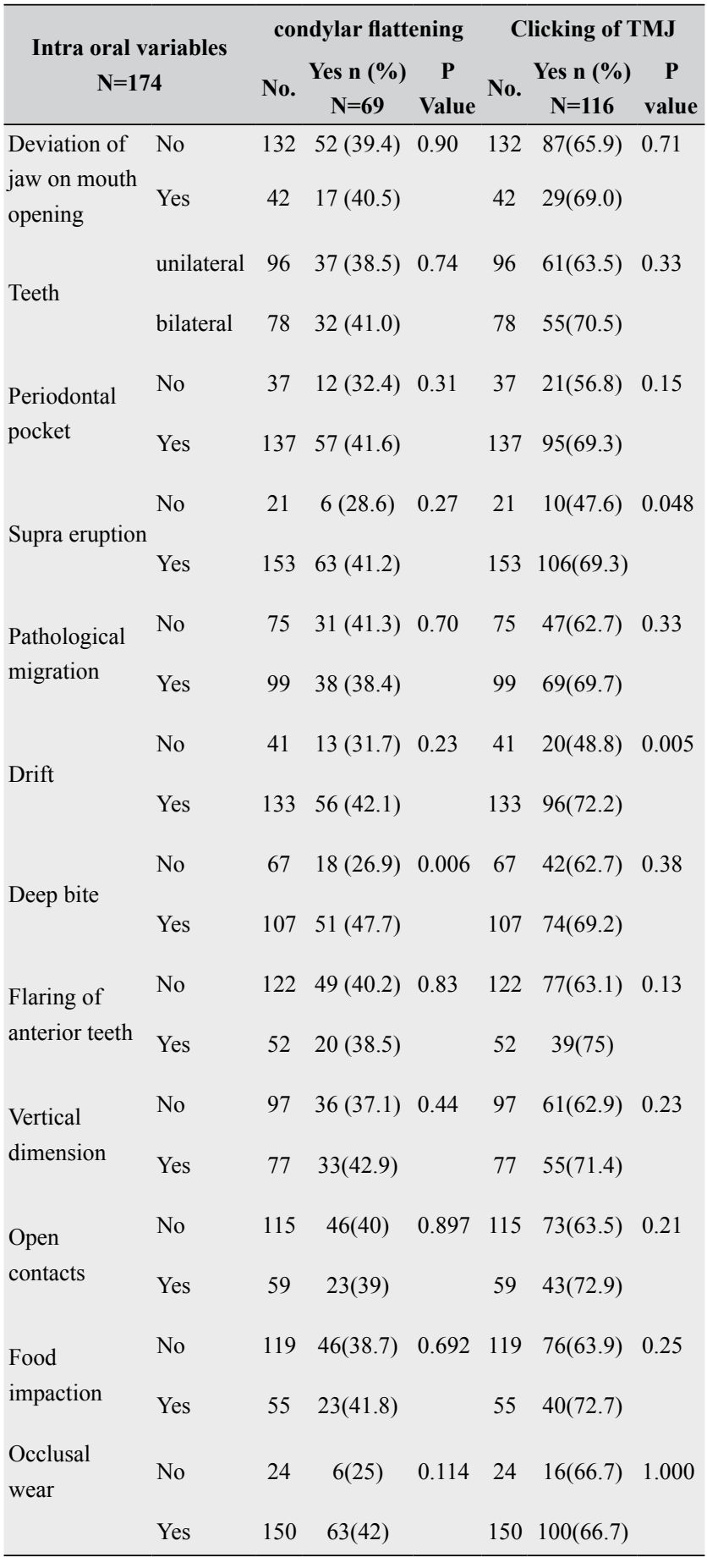

Another important observation made in the present study was the significant correlation of supraeruption of opposing molars and drifting of adjacent teeth, with clicking of the TMJ ( $p$ values being 0.048 and 0.005 respectively). Out of 174 patients, 153 patients had supraerupted teeth though clicking was observed in 106 patients. Drifting of adjacent teeth was also observed in a significantly large proportion, i.e., in 133 patients, out of which 96 patients had significant clicking. Furthermore, those patients with nonreplaced missing mandibular first molars for a duration of more than 3 years had significant clicking, with the $\mathrm{p}$ value being $0.007(\mathrm{p}<0.05)$ (Table II).

TABLE II: Clicking, Condylar flattening of TMJ and duration of missing molar (in months)

\begin{tabular}{|c|c|c|c|c|}
\hline & & $\begin{array}{c}\text { DURATION } \\
\text { (MONTHS) } \\
\text { Minimum, } \\
\text { maximum }\end{array}$ & $\begin{array}{c}\text { MEDIAN } \\
\left(Q_{1}, Q_{3}\right)\end{array}$ & P VALUE \\
\hline \multirow{2}{*}{ CLICKING } & YES & 2,480 & $\begin{array}{c}48 \\
(24,120)\end{array}$ & \multirow{2}{*}{0.007} \\
\hline & NO & 3,300 & $\begin{array}{c}24 \\
(24,54)\end{array}$ & \\
\hline \multirow{2}{*}{$\begin{array}{l}\text { CONDYLAR } \\
\text { FLATTENING }\end{array}$} & YES & 2,360 & $\begin{array}{c}48 \\
(24,108)\end{array}$ & \multirow{2}{*}{0.105} \\
\hline & NO & 3,480 & $\begin{array}{c}36 \\
(24,72)\end{array}$ & \\
\hline
\end{tabular}

However, the relationship between flattening of the condyles and duration of missing molars was not statistically significant $(\mathrm{p}>0.05)$.

\section{Discussion:}

Occlusion embraces the dynamic morphological and functional relationships between all components of the masticatory system - not just teeth and their supporting tissues but also the neuromuscular system, the temporomandibular joints (TMJs) and the craniofacial skeleton. ${ }^{3-6}$ Patient comfort and oral functionality depends on vertical dimension of teeth, occlusion, maintenance of hard tissue and temporomandibular joint health. Occlusal stability can be defined as the "stability of tooth positioning relative to its spatial relationship in the occluding dental arches". ${ }^{7}$ The occlusal instability could be attributed to changes like tooth migration, supraeruption, drifting, tilting of teeth and occlusal wear and often exacerbates in cases of unopposed and lone standing teeth with inadequate periodontal support. ${ }^{8}$ I In the present study the focus was on mandibular first molars as these are the 'key teeth in the dentition' owing to their position in the dental arch and their role in occlusion.

The paradox of the missing mandibular molar lies in the fact that in spite of being the first permanent teeth to erupt in the oral cavity, they are often the earliest to be lost and could thereby be left unreplaced for indefinite periods. In this study, we observed that the non-replacement of these missing first molars (unilateral /bilateral) resulted in changes in occlusion like drifting, supraeruption, deep bite, pathological migration of teeth, open contacts and food impaction in the adjacent teeth. We further observed the effects of these changes on the temporomandibular joint (TMJ). 
The symptoms of temporomandibular joint disorder (TMD) are facial pain, pain in the jaw joint and surrounding tissues, including the ear, jaw locking (open or closed), limited opening or inability to open the mouth comfortably, muscle spasms.9 The most common and frequently observed changes in the TMJ due to malocclusion, mentioned in literature, are clicking of TMJ9,10 and flattening of the condyles.11 Thus, in the present study, we determined TMJ dysfunction by assessing the clicking of the TMJ, deviation of the jaw on mouth opening and flattening of the condyles. Flattening of the condyles was assessed radiographically with orthopantomographs (OPG). OPG is suitable for the examination of condyles, though it reveals a lower level of involvement than tomography and MRI. It provides screening of bony structures of the condyles and it provides a broad coverage of the oral region for radiographic interpretation. $^{12}$

One of the observed changes due to the missing mandibular molar was deep bite. It is said to be one of the most damaging malocclusions which may adversely affect the periodontal support, occlusion and / or TMJ. In the current study, statistically significant association was found between deep bite and condylar flattening of the TMJ. In the sample size of 174 patients, a total of 107 patients had deep bite and 51 patients $(47.7 \%)$ with deep bite had radiographic changes of condylar flattening. A previous study ${ }^{5}$ done in 22 human skulls had demonstrated that deep bite resulted in asymmetrical occlusion, suggesting that asymmetry of occlusion and condyles are associated. Large rotation of the condyles in deep incisal bite cases has also been suggested as one of the causes of TMD. ${ }^{13}$ Eighteen patients with deep bite did not show significant changes in the condyle morphology. Forty nine patients, who did not demonstrate deep bite showed changes in the condylar shape and these changes can be attributed to other factors like muscular changes and anatomical factors as the molars are directly under the impact of the masticatory forces of the posterior vertical chain of muscles and this tends to be greater in case of a lone standing molar. When the muscle pull is strong and anteriorly positioned, a greater depressive action is transmitted to the dentition. ${ }^{7}$ Hence, it was concluded that deep bite can be one of the factors of malocclusion, resulting in changes in the morphology of the condyles of the TMJ.

In the present study, 153 patients had supraerupted opposing teeth and clicking of the TMJ was observed in 106 patients (69.3\%) of 153 patients. Drifting of teeth adjacent to the missing molars was also a frequent finding with 133 patients demonstrating these changes and clicking was observed in 96 patients $(72.2 \%)$ out of 133 patients. Supraeruption, mesial tilting, drifting of teeth are some of the factors resulting in malocclusion. It has been proposed that once the teeth begin to shift in angle, the vector of force tends to increase tooth tilting and thus imposing a different bio mechanical effect on the mandible. ${ }^{14}$ These results agree with concept of tightly locked occlusion, a kind of secondary change associated with supra eruption, drift or tipping, and tilting of the posterior teeth which is associated with TMD. ${ }^{15}$ Previous studies have also observed that TMJ sounds may originate from changes in articular surfaces, ${ }^{16,17}$ deviations in the form of articular components ${ }^{16,17}$ and lack of muscle coordination. ${ }^{18,19}$ In another study, it was observed that loss of molar support was involved in the initiation of lesions on the load bearing articular surfaces of the condyle and articular eminence of younger individuals. ${ }^{11}$ All of the mechanisms described above, can explain the increased prevalence of the joint sounds associated with supraeruption and drifting of teeth in the current study.

Yet another finding in the current study was that during clinical examination, clicking was observed in 61 patients with unilateral loss of molars and 55 patients with bilateral loss. Radiographic changes of condylar flattening were significant in 32 bilaterally missing molar patients and in 37 patients with unilateral loss of molars. This difference can be attributed to the fact that temporomandibular joint is a functional joint and an extension of the mandible. The functional pressure generated on the TMJ due to occlusion would be more in unilateral missing cases as compared to cases with bilateral missing molars. The concept of differential loading of the $\mathrm{TMJ}^{14}$ also explains this observation in the study. However, in the present study, the correlation between the above mentioned factors were found to be weak and not statistically significant.

It was also observed, that in cases of unilaterally missing molar, clicking was appreciated well on the opposite TM joint, as compared to the TM joint on the same side of the missing teeth. This can be considered as an adaptive remodeling or a compensatory adaptation of the opposing condyle to the changes occurring in the occlusion. However, the association was not found to be statistically significant. To the best of our knowledge, no literature for comparison of these factors is available.

A unique observation in the study was that duration of the missing mandibular molar was found to have an association with the changes in the TMJ. Eighty four patients in the study with the duration of missing molars ranging from 2 years 
to 10 years had demonstrated clicking. However condylar flattening of the TMJ did not demonstrate any significant relationship with the duration of tooth loss. There is no considerable evidence from the previous studies supporting this observation. Further studies might be required in this direction and probably more sensitive radiographs may help to reveal finer changes occurring in the condyles.

Also, factors like occlusal wear, presence of periodontal pocket in adjacent and opposing molars, flaring of anterior teeth, food impaction, which directly or indirectly result in occlusal instability, demonstrated a weak correlation with TMD. Deviation of jaw on mouth opening was observed in 37 patients amongst 174 patients with unilateral/ bilateral loss of molars and hence no statistically significant association could be elucidated. No statistically significant gender differences were noticed related to changes intraorally and their association with TMJ.

Few studies, ${ }^{20,21}$ reported a limited or no significant association between missing posterior teeth and $\mathrm{TMJ}$, while other studies ${ }^{11,15,22}$ reported a significant association between these two factors. A study by Barghi et al in 1992 has stressed on the impact of prosthetic rehabilitation on temporomandibular joint. ${ }^{23}$ It was observed that following replacement of posterior teeth, the clicking amplitude decreased in $68 \%$ of recordings, remained unchanged in $11 \%$, and increased in $21 \%$. Hence, in view of the various contradictory and supporting studies, it was inferred from the present study, that these malocclusions due to the nonreplaced mandibular molar may be viewed as a contributing factor, if not a causal factor in the pathogenesis of TMJ dysfunction.

\section{Conclusion:}

The present study has provided evidence of a statistically significant correlation between malocclusions resulting due to non-replacement of mandibular first molars and TMJ disorder. These pillars of occlusion, if lost and left unattended could shake the very foundation of stomatognathic stability. It was observed that missing mandibular molar space caused a cascade of untoward effects such as drifting, supra eruption and deep bite and in several cases culminated in temporomandibular dysfunction. A timely replacement of missing tooth may provide a resolution for preventing these gradual changes in the occlusal pattern, thereby maintaining the normalcy of TMJ function. Clinicians should be aware of the consequences of the non-replacement of these mandibular first molars. Moreover, patients should be informed and motivated to comply with replacement.

\section{Acknowledgements}

The authors wish to thank Dr. Richa Gupta (Senior Resident, PGIMER), Dr. Sanjana Ravi Kumar (Endodontist), Dr. Mihir Kulkarni, Dr. Pramod Krishna (post graduates) and Dr. Asif Mohammedali and Dr. Moosa Dalwai (Interns) of Manipal College of Dental Sciences, Manipal for their assistance.

\section{References:}

1) Mongini $F$. Influence of function on temporomandibular joint remodeling and degenerative disease. Dent Clin North Am 1983;27:479-94.

2) Wang MQ, He JJ, Chen CS,et al. A preliminary anatomical study on the association of condylar and occlusal asymmetry. Cranio 2011;29:111-6.

3) McNeill C. Fundamental treatment goals. In: McNeill C, ed. Science and practice of occlusion. Chicago, IL: Quintessence 1997:306-22.

4) Klineberg I, Jagger R. Occlusion and clinical practice An evidence based approach. Edinburgh: Wright, 2004.

5) Turp JC, Greene CS, Strub JR. Dental occlusion: a critical reflection on past, present and future concepts. Review Article. J Oral Rehabil 2008;35:446-53.

http://dx.doi.org/10.1111/j.1365-2842.2007.01820.x

6) Armellini D, Fraunhofer JA. The shortened dental arch: A review of the literature. J Prosthet Dent 2004;92:5315.

\section{http://dx.doi.org/10.1016/j.prosdent.2004.08.013}

7) Witter DJ, Creugers NH, Kreulen CM, et al. Occlusal stability in shortened dental arches. J Dent Res 2001;80:432-6.

\section{http://dx.doi.org/10.1177/00220345010800020601}

8) Sreedhar C, Baratam S. Deep overbite- A review (Deep bite, Deep overbite, and Excessive overbite). Annals and Essences of Dentistry 2009:8-25.

9) Okeson JP, de Leeuw R. Differential diagnosis of temporomandibular disorders and other orofacial pain disorders. Dent Clin North Am 2011;55:105-20.

http://dx.doi.org/10.1016/j.cden.2010.08.007

10) Pullinger AG, Seligman DA, Solberg WK. Temporomandibular disorders. Part II: Occlusal factors associated with temporomandibular joint tenderness and dysfunction. J Prosthet Dent 1988;59:363-7.

http://dx.doi.org/10.1016/0022-3913(88)90191-6

11) Wang MQ, Zhang M, Zhang JH. Photoelastic study of the effects of the occlusal surface morphology on tooth 
apical stress from vertical bite forces. J Contemp Dent Pract 2004;5:74-93.

12) Shashikumar HC, Durgekar SG, Nagaraj K, et al. Morphological study of temporomandibular joint in orthodontically treated patients by using pretreatment and post treatment orthopantomographs. The Orthodontic cyber Journal. 2011. http://orthocj. com/2011/12/5552/

13) Darendeliler N, Dinçer M, Soylu R. The biomechanical relationship between incisor and condylar guidances in deep bite and normal cases. J Oral Rehabil 2004;31:4307.

http://dx.doi.org/10.1111/j.1365-2842.2004.01264.x

14) Obrez A, GalloLM. Functional and biomechanical analysis of the TMJ.In: Laskin,DM,eds.

Temporomandibular disorders, an evidence based approach to diagnosis and treatment.IL: Quintessence 2006:29-30.

15) Wang MQ, Cao HT, Liu FR, et al. Association of tightly locked occlusion with temporomandibular disorders. J Oral Rehabil 2007;34:169-73.

\section{http://dx.doi.org/10.1111/j.1365-2842.2006.01657.x}

16) Nilner M. Relationships between oral parafunctions and functional disturbances and diseases of the stomatognathic system among children aged 714 years. Acta Odontol Scand 1983;41:167-72.

http://dx.doi.org/10.3109/00016358309162319

17) Razook SJ, Gotcher JE Jr, Bays RA. Temporomandibular joint noises in infants review of the literature and report of cases. Oral Surg Oral Med Oral Pathol 1989;67:65864.

http://dx.doi.org/10.1016/0030-4220(89)90004-2

18) Perry HT Jr. Adolescent temporomandibular joint dysfunction. Am J Orthod 1973;63:517-25.

http://dx.doi.org/10.1016/0002-9416(73)90164-4

19) Nilner M, Lassing SA. Prevalence of functional disturbances and diseases of stomatognathic system in 7-14 year olds. Swed Dent J 1981;5:173-87.

20) Seligman DA, Pullinger AG. Analysis of occlusal variables, dental attrition, and age for distinguishing healthy controls from female patients with intracapsular temporomandibular disorders. J Prosthet Dent 2000;83:76-82.

http://dx.doi.org/10.1016/S0022-3913(00)70091-6

21) Panula K. Correction of dentofacial deformities with orthognathic surgery Outcome of treatment with special reference to costs, benefits and risks. Department of Oral and Maxillofacial Surgery, University of Oulu Institute of Dentistry, University of Oulu.Thesis

22) Kirveskari P, Alanen P. Association between tooth loss and TMJ dysfunction. J Oral Rehabil 1985;12:189-94.

http://dx.doi.org/10.1111/j.1365-2842.1985.tb00635.x

23) Barghi N, dos Santos Junior J, Narendran S. Effects of posterior teeth replacement on temporomandibular joint sounds: A preliminary report. J Prosthet Dent 1992;68:132-6.

http://dx.doi.org/10.1016/0022-3913(92)90301-P 